\title{
Electron Microscopy Study of ALD Protective Coating on the FeOF Electrode
}

\author{
Chuan-Fu Lin ${ }^{1}$, Sz-Chian Liou ${ }^{2}$, Malachi Noked ${ }^{1}$, Wen-An Chiou ${ }^{2}$ and Gary Rubloff ${ }^{1,2}$ \\ ${ }^{1 .}$ Dept. of Materials Science and Eng., University of Maryland, College Park, MD, U.S.A. \\ 2. AIM Lab, NanoCenter, University of Maryland, College Park, MD, U.S.A
}

Rechargeable Li-ion batteries have become instrumental in powering portable electronics and battery electric vehicles due to their high energy density. The demand of developing next-generation higher energy density electrode materials has risen significantly in recent years. Iron fluoride $\left(\mathrm{FeF}_{3}\right)$ and iron oxyfluoride (FeOF) have been widely investigated as a positive electrode material because of their high theoretical capacity in the range of $500 \sim 800 \mathrm{mAh} / \mathrm{g}$ [1]. However, the metallic Fe and insulting LiF, formed through the conversion reaction during the discharge process results in poor reversibility and capacity retention [1]. To mitigate the degradation of the electrode materials upon cycling, a thin protective layer of solid electrolyte - lithium phosphor oxynitride (LiPON), via atomic layer deposition (ALD) was developed and applied on the FeOF particles [2, 3]. This study reports microstructure and chemical evolutions of FeOF with and without the protective thin films before and after discharge process using both electron microscopy and focused ion beam (FIB) technology.

The crystalline FeOF nanoparticles were synthesized by wet chemistry method and calcined at $\sim 200{ }^{\circ} \mathrm{C}$ [4]. FeOF nanoparticles were then mix with carbon black (CB) and polyvinylidene difluoride (PVDF) binder to form composites, and fabricated on the Al foil by tape-casting process. The LiPON coating was deposited on FeOF/CB composites by $\mathrm{ALD}[2,3]$. To minimize the exposure of $\mathrm{Li}$ in the environment, TEM thin specimens were prepared by FIB (Tescan GAIA) and quickly loaded onto TEM sample holder and inserted into TEM. TEM specimens were also prepared with an ultra-microtome (Reichert Ultracut E) for comparison. Microstructure and microchemistry were investigated using JEOL 2100 FEG TEM/STEM equipped with Gatan Tridiem 863 EELS/GIF and Oxford EDS systems. The distribution of constitute elements through LiPON/FeOF/CB composites was analyzed by time-of-flight secondary ion mass spectroscopy (ToF-SIMS) attached on Tescan FIB/SEM (GAIA).

SEM images of FeOF (open arrows) and CB/PVDF composites (arrows) clearly revealed morphological differences between specimens without and with LiPON coating (Figs. 1a and b, respectively). Depth profiles of $\mathrm{Li}^{+}, \mathrm{N}$ and $\mathrm{P}$ obtained from ToF-SIMS depicted that LiPON was well- deposited and penetrated into the FeOF/CB composite for $\sim 2 \mu \mathrm{m}$ (Fig. 1c). The TEM image revealed uniform and conformal coating of LiPON on FeOF particles by ALD (Fig. 2). Ultra-microtomed cross-sectional TEM images and selected area diffraction (SAD) patterns of LiPON coated FeOF/CB composite before discharge reaction showed FeOF particles with tetrahedron- and rod-like morphology mixed with round CB particles (Fig. 3). While TEM images and SAD patterns of bare LiPON on FeOF after discharge depicted drastic morphological change (both size and shape) of FeOF and the formation of irreversible crystalline Fe through conversion reaction (Fig. 4), LiPON protected FeOF composites after discharge were very similar to those before discharge reaction (Fig. 5). Sharp rings in SAD pattern indicate the crystalline $\mathrm{FeOF}(\mathrm{P} 42 / \mathrm{m} \mathrm{n} \mathrm{m}$ ) remained after lithiation. EELS spectrum and elemental mapping confirmed that the FeOF/CB electrode was well-protected by LiPON film. This study demonstrated the control of conversion reaction in electrode materials through ALD LiPON coating [5].

References:

[1] N. Pereira et. al., J. Electrochem. Soc., 156(6) (2009), A 407.

[2] A. C. Kozen et. al, Chem. Mater. 27 (2015), p. 5324.

[3] C-F Lin et al, (submitted for publication) (2017). 
[4] X. Fan et al, Nano Letters 15(11) (2015), p. 7650.

[5] Research was supported by Energy Frontier Research Center, DOE (DESC0001160) and UMD.

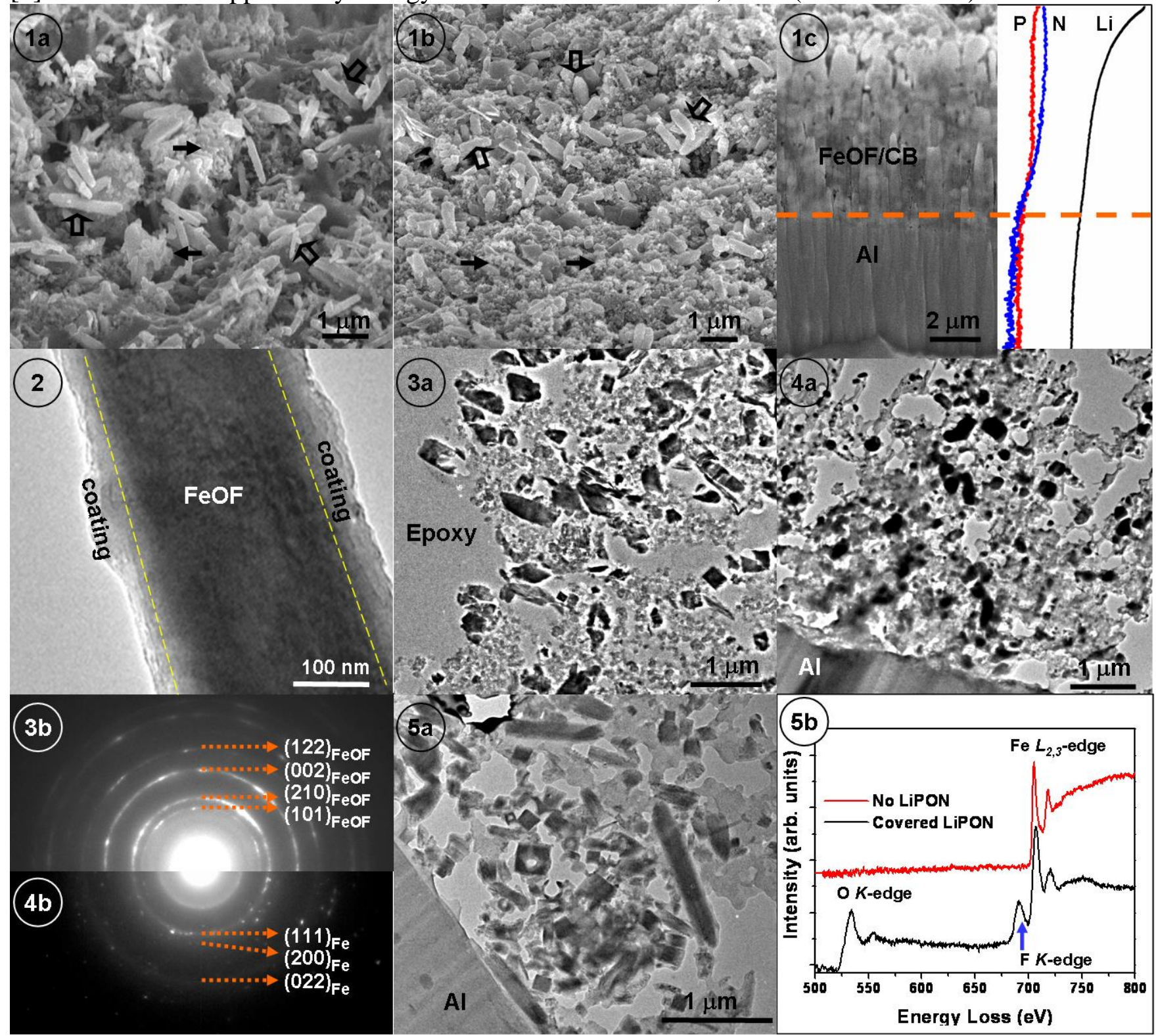

Figure 1. SEM images of FeOF/CB without LiPON (a) and with LiPON (b) coatings showed FeOF (rod-like, open arrows) mixed with CB (spherical, arrows) particles. With LiPON coating the surface of FeOF/CB composites appeared somewhat smooth (b). ToF-SIMS elemental depth profile revealed protective LiPON well-deposited into the FeOF/CB composites ( $2 \mu \mathrm{m}$ deep) (c).

Figure 2. TEM micrograph depicted uniform and conformal coating of LiPON on FeOF particles.

Figure 3. TEM image (a) and SAD pattern (b) of LiPON coated FeOF/CB composites (before discharge) depicted tetrahedron-/rod-like FeOF mixed with round/spherical CB particles.

Figure 4. TEM image (a) and SAD pattern (b) of FeOF/CB composites without LiPON coating (after discharge) showed drastic morphology change and formation of Fe after discharge reaction.

Figure 5. TEM image (a) and EELS spectrum (b) of LiPON coated FeOF/CB composites after discharge revealed unaltered morphology and chemical composition (as compared with Figs. 3 and 4). 